\title{
ON THE SOLUTIONS OF THE POLYNOMIC LAPLACIAN EQUATION
}

\author{
BY \\ GEORGE DASSIOS \\ Division of Applied Mathematics, Department of Chemical Engineering, University of Patras - and - \\ ICE/TH, FORTH, Patras, Greece

\begin{abstract}
In this short communication we demonstrate a representation of the solutions of a partial differential equation, which is a polynomial in the Laplacian, in terms of harmonic functions alone. The idea is based on the Vekua Trasformation, which connects the kernel of the Laplace operator with the kernel of the Helmholtz operator. The representation can be applied to some well-known equations, such as the Brinkman equation in Viscous Hydrodynamics or the equation of Shells in Elasticity.
\end{abstract}

Consider the polynomial

$$
P(t)=t^{n}+a_{n-1} t^{n-1}+a_{n-2} t^{n-2}+\cdots+a_{1} t+a_{0}
$$

where $a_{i}, i=0,1,2, \ldots, n-1$, are complex numbers, and the corresponding partial differential operator

$$
P(\Delta)=\Delta^{n}+a_{n-1} \Delta^{n-1}+a_{n-2} \Delta^{n-2}+\cdots+a_{1} \Delta+a_{0} .
$$

Let us assume that the polynomial (1) has the simple roots $\lambda_{1}, \lambda_{2}, \ldots, \lambda_{n}$. Then the operator (2) has the factorization

$$
P(\Delta)=\left(\Delta+\lambda_{1}\right)\left(\Delta+\lambda_{2}\right)\left(\Delta+\lambda_{3}\right) \cdots\left(\Delta+\lambda_{n}\right) .
$$

In the composition (3), to every nonzero root of (11) corresponds a Helmholtz operator, while to the zero root of (1) corresponds the Laplace operator.

Received November 20, 2015.

2010 Mathematics Subject Classification. Primary 35J05, 31C10, 31C99.

E-mail address: gdassios@chemeng.upatras.gr 
At this point we turn to the fundamental transform pair of Vekua [3], which in $\mathbb{R}^{3}$ assumes the form

$$
\begin{aligned}
u(\boldsymbol{r}) & =v(\boldsymbol{r})-\frac{k r}{2} \int_{0}^{1} v(t \boldsymbol{r}) J_{1}(k r \sqrt{1-t}) \sqrt{\frac{t}{1-t}} d t \\
v(\boldsymbol{r}) & =u(\boldsymbol{r})+\frac{k r}{2} \int_{0}^{1} u(t \boldsymbol{r}) I_{1}(k r \sqrt{t(1-t)}) \frac{1}{\sqrt{1-t}} d t
\end{aligned}
$$

where $\boldsymbol{r}$ refers to a star shape domain, $r=|\boldsymbol{r}|, J_{1}$ and $I_{1}$ stand for the Bessel and modified Bessel functions of the first degree, respectively, and $k$ is the constant of the Helmholtz operator $\left(\Delta+k^{2}\right)$. The essence of the Vekua transformation (4), (5) is the fact that if $v$ belongs to the kernel of the Laplace operator, then $u$ belongs to the kernel of the Helmholtz equation and vice versa.

Obviously, the most useful aspect of the transformation is the fact that it provides a solution of the Helmholtz equation

$$
\left(\Delta+k^{2}\right) u(\boldsymbol{r})=0
$$

in terms of a solution of the Laplace equation

$$
\Delta v(\boldsymbol{r})=0
$$

via equation (44). The $k$-dependence of the solution is always absorbed in the argument of the Bessel function. Furthermore, as we can observe in the relations (4) and (5) for $k=0$ the solutions $u$ and $v$ coincide.

Suppose now that

$$
\left(\Delta+\lambda_{k}\right) u_{k}(\boldsymbol{r})=0, \quad k=1,2,3, \ldots, n,
$$

and that each solution $u_{k}$ of the Helmholtz equation has the representation

$$
\begin{array}{r}
u_{k}(\boldsymbol{r})=F\left(v_{k}(\boldsymbol{r})\right)=v_{k}(\boldsymbol{r})-\frac{k r}{2} \int_{0}^{1} v_{k}(t \boldsymbol{r}) J_{1}(k r \sqrt{1-t}) \sqrt{\frac{t}{1-t}} d t \\
k=1,2,3, \ldots, n .
\end{array}
$$

Then, the general solution of equation

$$
P(\Delta) U(\boldsymbol{r})=0
$$

is represented by the $n$ harmonic functions $v_{k}$ as follows:

$$
U(\boldsymbol{r})=\sum_{k=1}^{n} F\left(v_{k}(\boldsymbol{r})\right)=\sum_{k=1}^{n} u_{k}(\boldsymbol{r})
$$


To prove this statement we apply successively from right to left the factors of the composition (3) to obtain

$$
\begin{aligned}
& \left(\Delta+\lambda_{1}\right)\left(\Delta+\lambda_{2}\right)\left(\Delta+\lambda_{3}\right) \cdots\left(\Delta+\lambda_{n-1}\right)\left(\Delta+\lambda_{n}\right)\left[u_{1}(\boldsymbol{r})+u_{2}(\boldsymbol{r})+u_{3}(\boldsymbol{r})+\cdots+u_{n}(\boldsymbol{r})\right] \\
& =\left(\Delta+\lambda_{1}\right)\left(\Delta+\lambda_{2}\right)\left(\Delta+\lambda_{3}\right) \cdots\left(\Delta+\lambda_{n-1}\right)\left[\left(\lambda_{n}-\lambda_{1}\right) u_{1}(\boldsymbol{r})+\left(\lambda_{n}-\lambda_{2}\right) u_{2}(\boldsymbol{r})\right. \\
& \left.+\left(\lambda_{n}-\lambda_{3}\right) u_{3}(\boldsymbol{r})+\cdots+\left(\lambda_{n}-\lambda_{n-1}\right) u_{n-1}(\boldsymbol{r})\right] \\
& =\left(\Delta+\lambda_{1}\right)\left(\Delta+\lambda_{2}\right)\left(\Delta+\lambda_{3}\right) \cdots\left(\Delta+\lambda_{n-2}\right)\left[\left(\lambda_{n}-\lambda_{1}\right)\left(\lambda_{n-1}-\lambda_{1}\right) u_{1}(\boldsymbol{r})\right. \\
& \left.+\left(\lambda_{n}-\lambda_{2}\right)\left(\lambda_{n-1}-\lambda_{2}\right) u_{2}(\boldsymbol{r})+\cdots+\left(\lambda_{n}-\lambda_{n-2}\right)\left(\lambda_{n-1}-\lambda_{n-2}\right) u_{n-2}(\boldsymbol{r})\right] \\
& =\cdots \\
& =\left(\Delta+\lambda_{1}\right)\left(\lambda_{n}-\lambda_{1}\right)\left(\lambda_{n-1}-\lambda_{1}\right)\left(\lambda_{n-2}-\lambda_{1}\right) \cdots\left(\lambda_{2}-\lambda_{1}\right) u_{1}(\boldsymbol{r}) \\
& =\left(\lambda_{n}-\lambda_{1}\right)\left(\lambda_{n-1}-\lambda_{1}\right)\left(\lambda_{n-2}-\lambda_{1}\right) \cdots\left(\lambda_{2}-\lambda_{1}\right)\left(\Delta+\lambda_{1}\right) u_{1}(\boldsymbol{r}) \\
& =0 .
\end{aligned}
$$

As a special case we consider the Brinkman equation [2]

$$
\left(\Delta^{2}+a \Delta\right) \Phi(\boldsymbol{r})=\Delta(\Delta+a) \Phi(\boldsymbol{r})=0,
$$

where the solution $\Phi$ accepts the representation

$$
\Phi(\boldsymbol{r})=v_{1}(\boldsymbol{r})+v_{2}(\boldsymbol{r})-\frac{k r}{2} \int_{0}^{1} v_{2}(t \boldsymbol{r}) J_{1}(k r \sqrt{1-t}) \sqrt{\frac{t}{1-t}} d t
$$

in terms of the two harmonic functions $v_{1}$ and $v_{2}$, where $k=\sqrt{a}$. Obviously, the harmonic function $v_{1}$ represents the factor $\Delta$ which corresponds to $\lambda=0$, while the rest of the representation (14) represents the factor $(\Delta+a)$.

Similarly the solution of the equation

$$
\left(\Delta^{2}+\beta \Delta+\gamma\right) \Psi(\boldsymbol{r})=\left(\Delta+\lambda_{1}\right)\left(\Delta+\lambda_{2}\right) \Psi(\boldsymbol{r})=0
$$

is represented as

$$
\begin{aligned}
\Psi(\boldsymbol{r}) & =v_{3}(\boldsymbol{r})-\frac{k_{1} r}{2} \int_{0}^{1} v_{3}(\operatorname{tr}) J_{1}\left(k_{1} r \sqrt{1-t}\right) \sqrt{\frac{t}{1-t}} d t \\
& +v_{4}(\boldsymbol{r})-\frac{k_{2} r}{2} \int_{0}^{1} v_{4}(\boldsymbol{t r}) J_{1}\left(k_{2} r \sqrt{1-t}\right) \sqrt{\frac{t}{1-t}} d t
\end{aligned}
$$


where

$$
\begin{gathered}
\Delta v_{3}(\boldsymbol{r})=\Delta v_{4}(\boldsymbol{r})=0, \\
\lambda_{1,2}=\frac{1}{2}\left(-\beta \pm \sqrt{\beta^{2}-4 \gamma}\right)
\end{gathered}
$$

and $k_{1}=\sqrt{\lambda_{1}}, k_{2}=\sqrt{\lambda_{2}}$.

Finally, we mention that the transformation (44), (5) holds for interior domains. For exterior domains we refer to 1 .

\section{REFERENCES}

[1] Antonios Charalambopoulos and George Dassios, On the Vekua pair in spheroidal geometry and its role in solving boundary value problems, Appl. Anal. 81 (2002), no. 1, 85-113, DOI 10.1080/0003681021000021088. MR:1926803

[2] D. Palaniappan, On some general solutions of transient Stokes and Brinkman equations, Journal of Theoretical and Applied Mechanics 52 (2014), 405-415.

[3] I. N. Vekua, New methods for solving elliptic equations, translated from the Russian by D. E. Brown, translation edited by A. B. Tayler, North-Holland Series in Applied Mathematics and Mechanics, Vol. 1, North-Holland Publishing Co., Amsterdam; Interscience Publishers John Wiley \& Sons, Inc., New York, 1967. MR0212370 